\title{
DNA variation in the metallothionein genes in wild rice Oryza rufipogon: Relationship between DNA sequence polymorphism, codon bias and gene expression
}

\author{
Naohiko T. Miyashita ${ }^{1}$, Kentaro Yoshida $^{1}$ and Takashige Ishii ${ }^{2}$ \\ ${ }^{1}$ Laboratory of Plant Genetics, Graduate School of Agriculture, Kyoto University, Sakyo-ku, \\ Kyoto 606-8502 Japan \\ ${ }^{2}$ Laboratory of Plant Breeding, Faculty of Agriculture, Kobe University, \\ Nada-ku, Kobe 657-8501 Japan
}

(Received 19 May 2005, accepted 4 July 2005)

\begin{abstract}
This study examines the relationship between DNA sequence variation and level of gene expression in four metallothionein genes from wild rice Oryza rufipogon. The nucleotide diversity was 0.0028 to 0.0117 over the entire coding and non-coding region, and it was negatively correlated with gene expression for three type 2 metallothionein genes. In contrast, codon bias and percent of preferred codons correlated positively with gene expression. These results indicate that the intensity of natural selection depends on the level of gene expression, which in turn shapes the level of nucleotide polymorphism. In addition, significant linkage disequilibria were frequent between the metallothionein genes, although significance was not confirmed after multiple test correction. This result suggests that metallothionein genes expressed at different levels are epistatic with respect to fitness, and that gene expression is an important factor determining level of DNA polymorphism.
\end{abstract}

Key words: DNA polymorphism, gene expression, codon bias, metallothionein, wild rice Oryza rufipogon

\section{INTRODUCTION}

Natural selection is one of the most important genetic mechanisms that determine the level of DNA sequence polymorphism in natural populations (Kimura 1983). Simply speaking, the degree of selection pressure correlates inversely with the amount of DNA sequence polymorphism (i.e., stronger selective pressure, less DNA sequence polymorphism) although other mechanisms such as genetic drift and recombination also influence the amount of DNA sequence variation in a specific gene or genomic region. In many species such as Drosophila (Moriyama and Powell 1996) and A. thaliana (Yoshida et al. 2003; Miyashita 2003), the level of nucleotide variation is not constant throughout the genome, but varies for different genes or gene regions, which may reflect the difference in functional importance of different gene products.

The level of gene expression is one aspect of the functional importance of a particular gene. If the level of gene expression is positively correlated with functional importance, then we expect to observe that highly expressed genes will have a lower level of polymorphism

Edited by Fumio Tajima

* Corresponding author: E-mail: arabis@kais.kyoto-u.ac.jp than weakly expressed genes. To examine this expectation, the exact measurement of gene expression of several loci with the same function is necessary. Even if level of gene expression differs among a set of loci of different functions, it is not possible to test the correlation, because difference in functional importance might contribute to difference in level of DNA polymorphism. An excellent opportunity to execute this kind of test was provided by the work of SAGE (serial analysis of gene expression: Velculescu et al. 1995) on Oryza sativa (Matsumura et al. 1999), where they showed that several metallothionein genes were expressed at different levels in rice seedlings.

Metallothionein (MT) is a small cysteine (Cys)-rich protein found in fungi, animals and plants, whose proposed function is detoxification of heavy metals, such as cadmium, zinc and copper (Kägi and Schäffer 1988). Genes encoding MT and MT-like proteins have been identified in many plant species including wheat (Kawashima et al. 1992), barley (Okumura et al. 1991), and Arabidopsis (Zhou and Goldsbrough 1995). It was reported that higher abundance of MT mRNA correlates with higher tolerance to heavy metals in Arabidopsis (Murphy and Taiz 1995). As in other organisms, MT genes in rice consist of a multigene family (Hsieh et al. 1995), and many 
ESTs of MT genes have been detected in rice EST database. Considering abundant expression of MT genes in rice seedlings under normal growth conditions, Mutsumura et al. (1999) speculated that MTs are not solely involved in detoxification, but also play an important function during plant growth such as transport of metal cofactors or reduction of free metal ions in cells.

In this study, DNA polymorphism in four metallothionein genes differentially expressed in rice seedlings was examined for the wild rice $O$. rufipogon, which is the most closely related ancestral species of $O$. sativa (Oka and Chang 1959). These species are morphologically similar to each other, and occur in ecologically similar conditions (Morishima et al. 1984). The gene structure and nucleotide sequence variation for the MT genes and Adh1 (Yoshida et al. 2004) are indistinguishable between $O$. sativa and $O$. rufipogon. These observations suggest that their gene expression profiles might be identical.

The primary goal of this study is to examine the relationship between gene expression and DNA polymorphism. In addition, codon bias, which is positively correlated with gene expression in other organisms (Grantham et al. 1981; Ikemura 1981), is also examined. Furthermore, gene expression in living organisms must be under coordinated regulation mechanism (Cooper 2000). Different levels of gene expression among the MT genes could be a consequence of interaction between these genes and regulatory factors, which can be regarded as epistasis between the MT genes. Since epistasis is an important mechanism contributing to linkage disequilibrium in natural populations (Kimura 1956), non-random association between DNA polymorphism within and between these MT genes is examined. The second goal is to investigate the level and pattern of DNA polymorphism in nuclear genes of Oryza species, a model monocot plant species. It is important to gather information on the level and pattern of DNA polymorphism of diverse organisms to clarify genetic and evolutionary mechanisms of organic evolution.

\section{MATERIALS AND METODS}

Plant materials. From 11 to 16 accessions of $O$. rufipogon, sampled in China and Southeast Asia, and one accession of $O$. barthii, were used in this study (Table 1). These species and $O$. sativa commonly have the A genome. Growth conditions of these plants and procedure for extraction of total DNA were described in Yoshida et al. (2004).

Metallothionein genes investigated. Matsumura et al. (1999) reported the top 30 highest abundance transcripts in rice seedlings. Four MT genes were included in this group and three additional MT genes were identified in their study (personal communication by $\mathrm{H}$. Matsumura and R. Terauchi, Iwate Biotechnology Research

Table 1 Plant materials used in this study

\begin{tabular}{lllcccc}
\hline \hline Species & Line & Origin & met1 & met2 & met5 & met6 \\
\hline Oryza rufipogon & W108 & India & $\bigcirc$ & $\bigcirc$ & $\bigcirc$ & $\bigcirc$ \\
& W593 & Malaysia & $\bigcirc$ & $\bigcirc$ & $\bigcirc$ & $\bigcirc$ \\
& W120 & India & $\bigcirc$ & $\bigcirc$ & $\bigcirc$ & $\bigcirc$ \\
& W149 & India & $\bigcirc$ & $\bigcirc$ & $\bigcirc$ & \\
& W630 & Myanmar & $\bigcirc$ & $\bigcirc$ & $\bigcirc$ & $\bigcirc$ \\
& W1956 & China & $\bigcirc$ & $\bigcirc$ & $\bigcirc$ & $\bigcirc$ \\
& W1962 & China & $\bigcirc$ & & $\bigcirc$ & \\
& W1964 & China & $\bigcirc$ & & $\bigcirc$ & \\
& W1965 & China & $\bigcirc$ & & $\bigcirc$ & \\
& W1967 & China & $\bigcirc$ & & $\bigcirc$ & \\
& W1972 & Indonesia & $\bigcirc$ & & $\bigcirc$ & $\bigcirc$ \\
W1976 & Indonesia & $\bigcirc$ & $\bigcirc$ & $\bigcirc$ & $\bigcirc$ \\
& CT51 & Vietnam & & $\bigcirc$ & & $\bigcirc$ \\
CT56 & Vietnam & & & $\bigcirc$ & $\bigcirc$ \\
YG2A & Myanmar & & $\bigcirc$ & $\bigcirc$ & $\bigcirc$ \\
CB22 & Cambodia & & & $\bigcirc$ & $\bigcirc$ \\
LV61 & Laos & & $\bigcirc$ & & $\bigcirc$ \\
SN & Thailand & & $\bigcirc$ & $\bigcirc$ & $\bigcirc$ \\
W607 & Guinea & $\bigcirc$ & $\bigcirc$ & $\bigcirc$ & \\
\hline
\end{tabular}

Accessions used for each MT gene are marked. 
Table 2 Metallothionein genes analyzed in this study

\begin{tabular}{|c|c|c|c|c|c|}
\hline Rank & Accession $\#^{\mathrm{a}}$ & \#tag ${ }^{\mathrm{b}}$ & code & chromosome $^{\mathrm{c}}$ & size (coding region) \\
\hline 1 & U57638 & 128 & met1 & $5 \mathrm{~S}(6.5 \mathrm{cM})$ & $1236 \mathrm{bp}(255 \mathrm{bp})$ \\
\hline 2 & AF001396 & 72 & met2 & $1 \mathrm{~S}(28.4 \mathrm{cM})$ & 909 bp (189 bp) \\
\hline 5 & AF017365 & 17 & met5 & $1 \mathrm{~L}(181.8 \mathrm{cM})$ & $656 \mathrm{bp}(243 \mathrm{bp})$ \\
\hline 6 & D89931 & 12 & met6 & $1 \mathrm{~S}(12.2 \mathrm{cM})$ & $1470 \mathrm{bp}(249 \mathrm{bp})$ \\
\hline
\end{tabular}

${ }^{a}$ : Accession \# in DNA database.

b: \# of tag detected among 10,122 tags analyzed in rice seedling (Matsumura et al. 1999).

c: location in $O$. sativa. Distance from tip of short arm is shown in parenthesis.

\section{met1 (chromosome 5)}

$1236 \mathrm{bp}$ (255bp coding region)

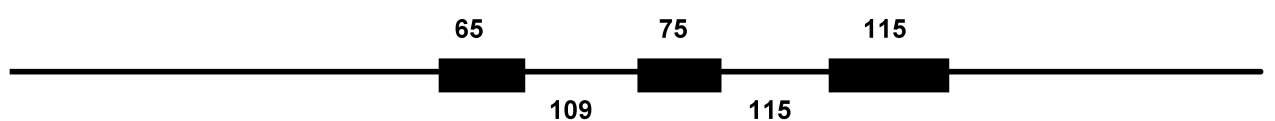

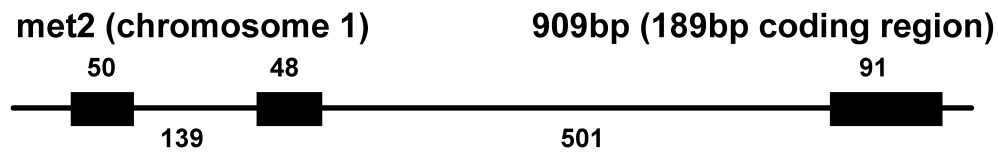

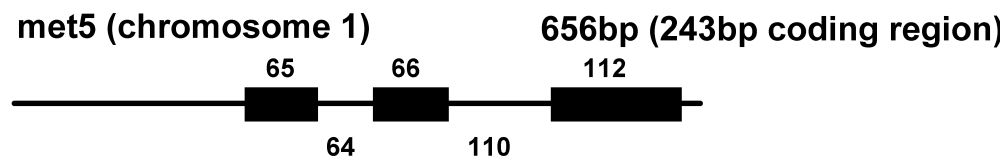

met6 (chromosome 1)

$$
65
$$

$1459 b p$ (249bp coding region)
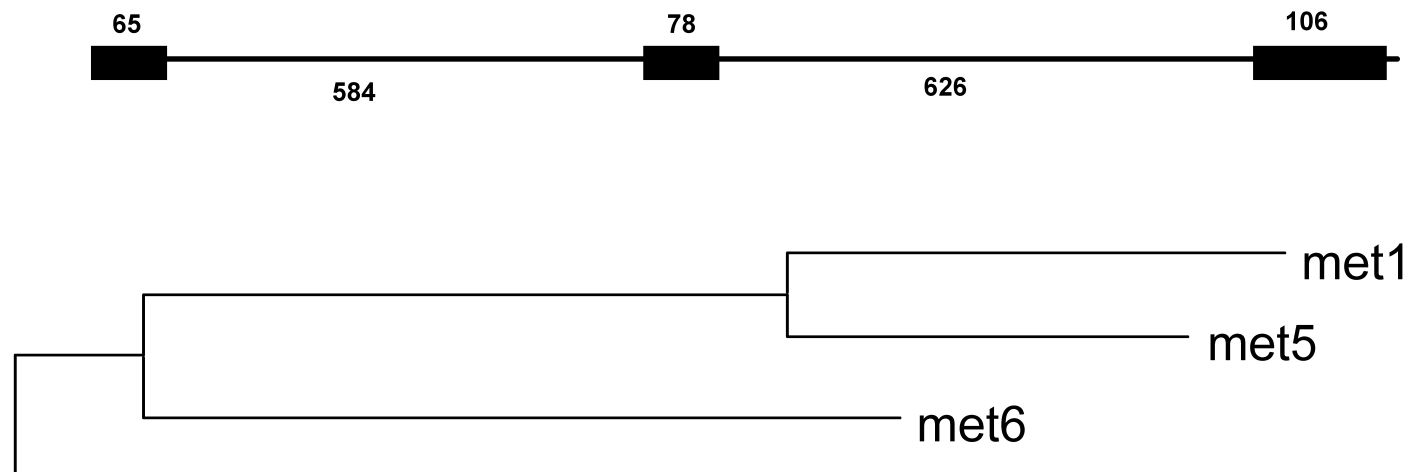

met2

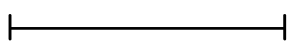

\section{1}

Fig. 1. Metallothionein genes investigated in this study. A) Exon-intron structure in the PCR amplified region of the four genes studied. Exon is indicated as a filled box. Nucleotide length of exon and intron is shown above and below the regions, respectively. B) Comparison of amino acid sequences of the four genes. Cystein residues typically found in the Type II metallothionein are shown at the bottom of the comparison. C) NJ tree based on amino acid sequence variations among the four genes.

Center, Kitakami, Iwate, Japan). A BLAST search against a rice database identified seven MT EST sequences and four nuclear genomic regions (Table 2). The rice MT genes were designated met1, met2, met5 and met6, according to rank of gene expression. One MT gene is on the $5^{\text {th }}$ chromosome and the others are on the $1^{\text {st }}$ chromosome of $O$. sativa. It was assumed that the MT genes have the same chromosomal location in $O$. rufipogon and $O$. sativa. All MT genes have three exons (Fig. 1) and the number of amino acids in the MT coding sequence ranges from 62 to 84 . In rice seedlings, the met1 is the most abundantly expressed gene among all 
the genes investigated (Matsumura et al. 1999). There is approximately 10 times difference in the level of gene expression (the number of tags detected) between the met 1 of high expression and met 6 of low expression. The amino acid sequence of met2 differs from the other three protein sequences, which appear to be typical Type 2 MT proteins (Robinson et al. 1993). This suggests that met2 may have distinct function.

Nucleotide sequence determination. MT genes were amplified by PCR. The amplified region was approximately $1.2,0.9,0.6$ and $1.4 \mathrm{~kb}$ for met1, met2, met5 and met6, respectively (Fig. 1). Primers were based on the $O$. sativa genomic DNA sequence. Nucleotide sequences of these primers will be provided upon request. PCR products were cloned into the plasmid pUC18 and their DNA sequence determined as described in Miyashita (2003). Newly determined sequences were deposited in the DDBJ databank under accession numbers AB175784$\mathrm{AB} 175808$, and AB175896-AB175925.
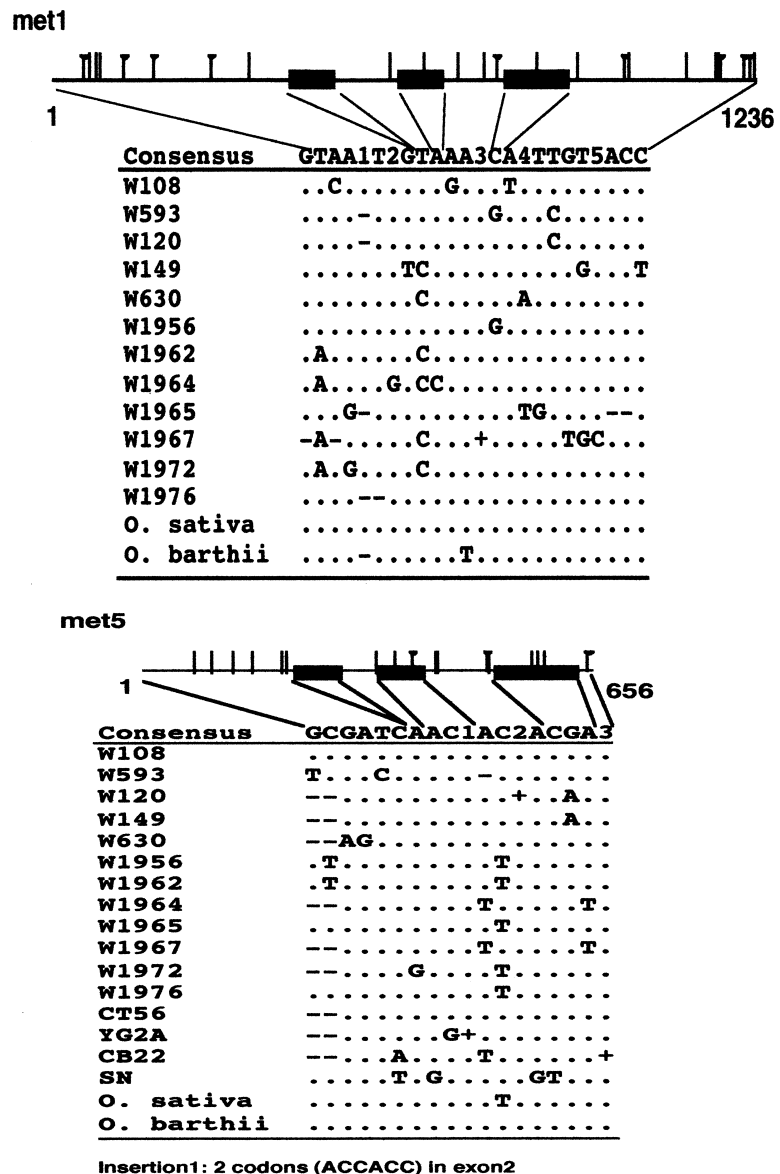

Fig. 2. Summary of DNA variations in metallothionein genes in Oryza. All the variations detected including singletons are shown. Numbers in the consensus sequence mean indel variations. Nucleotide sequences not shown in the summary of each gene are available upon request.
Data analyses. DNA sequence analysis was carried out using DnaSP version 3.5 (Rozas and Rozas 1999). Nucleotide diversity $(\pi)$ and $\theta(4 \mathrm{Ne} \mu$ : Watterson 1975) were estimated as the measures of nucleotide polymorphism. Tests of Tajima (Tajima 1989), and Fu and Li (Fu and $\mathrm{Li}$ 1993) were conducted to examine departure from neutrality. As a measure of codon usage bias, the effective number of codons (ENC: Wright 1990) was estimated. Percentage of the preferred codons (Akashi and Schaeffer 1997) in the MT genes was determined based on Kawabe and Miyashita (2003). Linkage disequilibrium between DNA sequence variants (site and indel) was examined using Chi-square test. NJ trees were constructed with MEGA (Kumar et al. 2001), based on genetic distance estimated for the entire region of each gene by Kimura's two-parameter method (Kimura 1980).

\section{RESULTS}

DNA polymorphism in $O$. rufipogon MT genes. DNA sequence polymorphism in four O. rufipogon MT
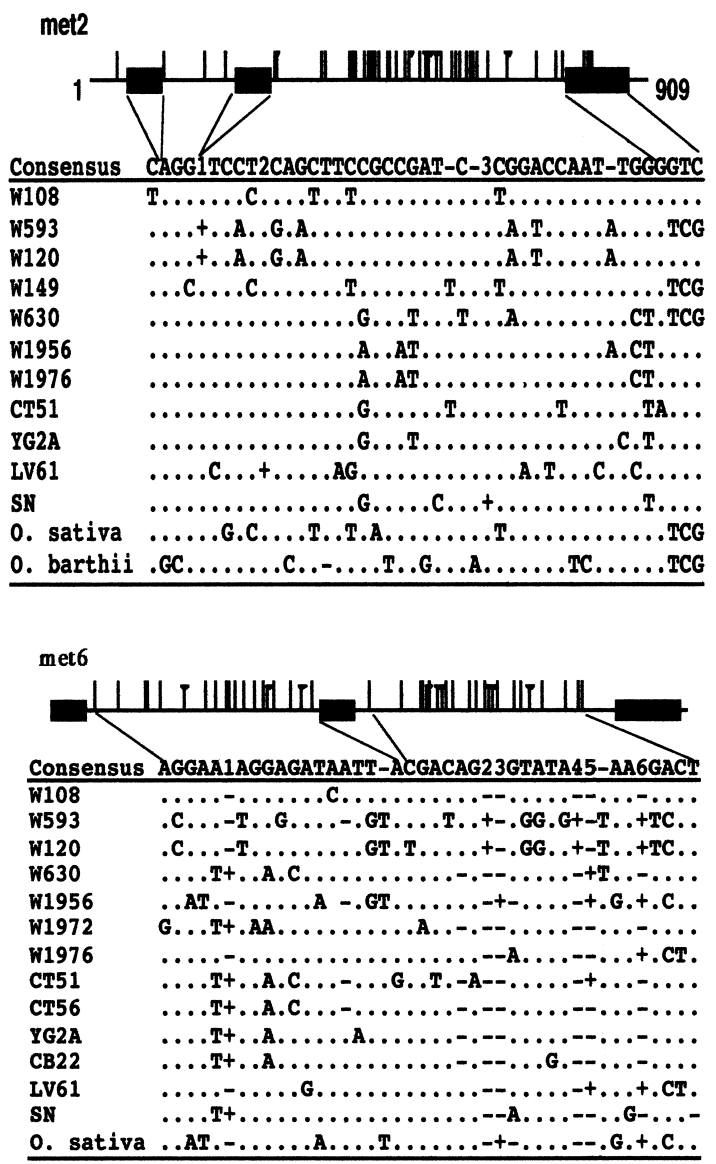
genes is summarized in Fig. 2. Each gene has a unique pattern of site changes and indels and no particular pattern is common to all genes. This is unlike the situation in A. thaliana, where dimorphism is observed in many nuclear genes(Innan et al. 1996; Yoshida et al. 2003). The met5 gene has a two codon insertion in the 2nd exon. No variation was detected in the coding region of met6. It is not possible to distinguish $O$. sativa (or $O$. barthii) and $O$. rufipogon by DNA variations detected in these MT genes. These species share most of nucleotide variations, although there are some species-specific variations. This result is consistent with the hypothesis that $O$. sativa was originated from $O$. rufipogon (Oka and Chang 1959).

The level of nucleotide variation was estimated for each MT gene and for functionally distinct gene regions (Table 3 ). For the entire gene, nucleotide diversity ranges from 0.0028 to 0.0101 . Met2 shows slightly higher nucleotide diversity than the other three MT genes, but not outside the range observed for nuclear genes in other plant species. The functionally distinct gene regions did not have consistently higher or lower nucleotide diversity and significant departure from neutrality was not detected, except for the 5' flanking region of met5 (Table 3). Test statistics were consistently negative, suggesting weak purifying selection on these genes.

Codon bias in O. rufipogon MT genes. Codon usage in the MT genes is shown in Table 4. O. rufipogon MTs have a high cysteine content, which has also been noted for MTs from other plant species (Kägi and Schäffer 1988), and because MT is a small protein, some amino acids (for example, Trp and Arg) are not used at all. In general, codon usage favors preferred codons, but fre- quencies of preferred codons vary in the four MT genes analyzed here.

The GC content is high in the MT coding region and at the $3^{\text {rd }}$ codon position (Table 5 ), which has been observed as a general property of nuclear genes in monocot (Poaceae) plants (Kawabe and Miyashita 2003). The average ENC (39.09) for the four MT genes is lower than the average ENC of nuclear genes of $O$. sativa (ibid.). This relatively high codon bias is probably due to the high frequency of preferred codons and limited number of amino acids used. Codon bias (ENC) and the frequency of preferred codons are higher in highly expressed MT genes (met1 and met2) than in weakly expressed MT genes (met5 and met6) (Table 5). This indicates that increasing codon bias and increasing use of preferred codons correlates with a higher level of gene expression in MT genes in O. rufipogon. This result is consistent with studies in bacteria (Grantham et al. 1981), Drosophila (Moriyama and Hartl 1993), tobacco, tomato and barley (Perlak et al. 1991; Jensen et al. 1996).

\section{Relationship between level of nucleotide variation and gene expression in $O$. rufipogon MT genes.}

Met2 has the highest level of nucleotide variation among the four MT genes analyzed in this study (Table 6), but it is more highly expressed than met5 and met6. However, it is possible that met2 is not a type $2 \mathrm{MT}$ and it might have a distinct function from the other three MT genes. When met2 is excluded and met1, met5 and met6 are analyzed, nucleotide diversity and level of gene expression are negatively correlated (Fig. 3) (i.e., higher expression corresponds to less nucleotide variation). Also, there is an almost perfect association between level of nucleotide variation for the entire region including introns and

Table 3 Level of nucleotide polymorphism in the metallothionein genes in Oryza rufipogon

\begin{tabular}{|c|c|c|c|c|c|c|c|c|c|c|c|c|c|c|c|c|}
\hline \multirow[b]{2}{*}{ Region } & \multicolumn{4}{|c|}{$\operatorname{met} 1(12)^{\mathrm{a}}$} & \multicolumn{4}{|c|}{ met2 (11) } & \multicolumn{4}{|c|}{ met5 (16) } & \multicolumn{4}{|c|}{ met6 (13) } \\
\hline & Size & $\mathrm{S}$ & $\begin{array}{c}\pi \\
\text { (Tajima'D }\end{array}$ & $\begin{array}{c}\theta \\
\text { Fu \& Li D) }\end{array}$ & Size & $\mathrm{S}$ & $\begin{array}{c}\pi \\
\text { (Tajima'D }\end{array}$ & $\begin{array}{c}\theta \\
\mathrm{Fu} \& \mathrm{Li} \mathrm{D})\end{array}$ & Size & $\mathrm{S}$ & $\begin{array}{c}\pi \\
\text { (Tajima'D }\end{array}$ & $\begin{array}{c}\theta \\
\text { Fu \& Li D) }\end{array}$ & Size & $\mathrm{S}$ & $\begin{array}{c}\pi \\
\text { (Tajima'D }\end{array}$ & $\begin{array}{c}\theta \\
u \& \mathrm{Li} \mathrm{D})\end{array}$ \\
\hline \multirow[t]{2}{*}{ Entire } & 1231 & 13 & 0.0028 & 0.0035 & 915 & 29 & 0.0101 & 0.0117 & 661 & 11 & 0.0039 & 0.0068 & 1474 & 30 & 0.0051 & 0.0070 \\
\hline & & & $(-0.88 \mathrm{NS}$ & $-0.73 \mathrm{NS})$ & & & $(-0.64 \mathrm{NS}$ & $-0.32 \mathrm{NS}$ ) & & & $(-1.62 \mathrm{NS}$ & $-1.95 \mathrm{NS})$ & & & $(-1.22 \mathrm{NS}$ & $-1.30 \mathrm{NS})$ \\
\hline \multirow[t]{2}{*}{ 5' flanking } & 407 & 3 & 0.0024 & 0.0024 & 60 & 1 & 0.0030 & 0.0057 & 216 & 4 & 0.0054 & 0.0131 & 0 & - & - & - \\
\hline & & & $(-0.13 \mathrm{NS}$ & $0.09 \mathrm{NS})$ & & & $(-1.13 \mathrm{NS}$ & $-1.29 \mathrm{NS})$ & & & $\left(-1.95^{*}\right.$ & $\left.-2.56^{*}\right)$ & & & (na & na) \\
\hline \multirow[t]{2}{*}{ Exons } & 255 & 2 & 0.0019 & 0.0026 & 189 & 4 & 0.0079 & 0.0072 & 249 & 5 & 0.0035 & 0.0062 & 249 & 0 & 0 & 0 \\
\hline & & & $(-0.85 \mathrm{NS}$ & $-0.37 \mathrm{NS})$ & & & $(0.34 \mathrm{NS}$ & $0.41 \mathrm{NS})$ & & & $(-1.46 \mathrm{NS}$ & $-1.05 \mathrm{NS})$ & & & (na & na) \\
\hline \multirow[t]{2}{*}{ Silent } & 56.8 & 1 & 0.0053 & 0.0058 & 37.6 & 0 & 0 & 0 & 56.1 & 3 & 0.0067 & 0.0161 & 56 & 0 & 0 & 0 \\
\hline & & & $(-0.20 \mathrm{NS}$ & $0.75 \mathrm{NS})$ & & & (na & na) & & & $(-1.70 \mathrm{NS}$ & $-2.20 \mathrm{NS})$ & & & (na & na) \\
\hline \multirow[t]{2}{*}{ Repl. } & 195.2 & 1 & 0.0009 & 0.0017 & 148.4 & 4 & 0.0101 & 0.0092 & 183.9 & 2 & 0.0025 & 0.0033 & 192 & 0 & 0 & 0 \\
\hline & & & $(-1.14 \mathrm{NS}$ & $-1.33 \mathrm{NS})$ & & & $(0.34 \mathrm{NS}$ & $0.41 \mathrm{NS})$ & & & $(-0.58 \mathrm{NS}$ & $0.91 \mathrm{NS})$ & & & (na & na) \\
\hline \multirow[t]{2}{*}{ Introns } & 225 & 2 & 0.0032 & 0.0030 & 630 & 24 & 0.0118 & 0.0141 & 174 & 2 & 0.0039 & 0.0038 & 1210 & 30 & 0.0065 & 0.0085 \\
\hline & & & $(0.22 \mathrm{NS}$ & $-0.37 \mathrm{NS})$ & & & $(-0.74 \mathrm{NS}$ & $-0.36 \mathrm{NS})$ & & & $(0.10 \mathrm{NS}$ & $-0.50 \mathrm{NS})$ & & & $(-1.03 \mathrm{NS}$ & $-0.77 \mathrm{NS})$ \\
\hline \multirow[t]{2}{*}{ 3' flanking } & 348 & 6 & 0.0037 & 0.0058 & 23 & 0 & 0 & 0 & 25 & 0 & 0 & 0 & 15 & 0 & 0 & 0 \\
\hline & & & $(-1.371 \mathrm{NS}$ & $-1.108 \mathrm{NS})$ & & & (na & na) & & & (na & na) & & & (na & na) \\
\hline
\end{tabular}

a \# of samples, S: \# of segregating sites, NS: non-significant, * significant at $5 \%$ level, na: not applied. 
Table 4 Codon usage in the four MT genes in O. rufipogon

\begin{tabular}{|c|c|c|c|c|c|c|c|c|c|c|c|c|c|c|c|c|c|c|c|c|c|c|c|}
\hline & & \multicolumn{4}{|c|}{ met } & & & \multicolumn{4}{|c|}{ met } & & & \multicolumn{4}{|c|}{ met } & & & \multicolumn{4}{|c|}{ met } \\
\hline & & 1 & 2 & 5 & 6 & & & 1 & 2 & 5 & 6 & & & 1 & 2 & 5 & 6 & & & 1 & 2 & 5 & 6 \\
\hline \multirow[t]{2}{*}{ Phe } & UUU & 0 & 0 & 0 & 0 & Ser & UCU & 2 & 0 & 2 & 0 & Tyr & UAU & 0 & 1 & 0 & 0 & Cys & UGU & 1 & 1 & 0 & 0 \\
\hline & $U U U$ & 2 & 1 & 1 & 0 & & $U C C$ & 3 & 1 & 3 & 2 & & $U A C$ & 0 & 0 & 1 & 1 & & $U G C$ & 16 & 9 & 17 & 14 \\
\hline \multirow[t]{2}{*}{ Leu } & UUA & 0 & 0 & 0 & 0 & & UCA & 0 & 0 & 1 & 0 & stop & UAA & - & - & - & - & stop & UGA & - & - & - & - \\
\hline & $U U G$ & 0 & 0 & 0 & 1 & & $U C G$ & 0 & 1 & 1 & 1 & stop & UAG & - & - & - & - & $\operatorname{Trp}$ & UGG & 0 & 0 & 0 & 0 \\
\hline \multirow[t]{4}{*}{ Leu } & CUU & 0 & 0 & 1 & 0 & Pro & $\mathrm{CCU}$ & 1 & 0 & 0 & 1 & His & CAU & 0 & 0 & 0 & 1 & Arg & CGU & 0 & 0 & 0 & 0 \\
\hline & $C U C$ & 1 & 0 & 0 & 0 & & $C C C$ & 0 & 1 & 1 & 1 & & $C A C$ & 0 & 1 & 0 & 0 & & $C G C$ & 0 & 0 & 0 & 0 \\
\hline & CUA & 0 & 0 & 0 & 0 & & CCA & 1 & 0 & 0 & 0 & Gln & $\mathrm{CAA}$ & 0 & 0 & 0 & 0 & & CGA & 0 & 0 & 0 & 0 \\
\hline & $C U G$ & 0 & 0 & 0 & 0 & & $C C G$ & 0 & 0 & 0 & 1 & & $C A G$ & 1 & 1 & 1 & 2 & & CGG & 0 & 0 & 0 & 0 \\
\hline \multirow[t]{3}{*}{ Ile } & AUU & 0 & 0 & 0 & 0 & Thr & $\mathrm{ACU}$ & 0 & 0 & 0 & 2 & Asn & $\mathrm{AAU}$ & 0 & 0 & 0 & 0 & Ser & AGU & 0 & 0 & 0 & 0 \\
\hline & $A U C$ & 0 & 0 & 0 & 1 & & $A C C$ & 5 & 3 & 6 & 3 & & $A A C$ & 5 & 2 & 7 & 5 & & $A G C$ & 5 & 4 & 3 & 1 \\
\hline & AUA & 0 & 1 & 0 & 0 & & ACA & 1 & 0 & 0 & 0 & Lys & $\mathrm{AAA}$ & 0 & 1 & 0 & 0 & Arg & AGA & 0 & 0 & 0 & 0 \\
\hline Met & AUG & 4 & 1 & 2 & 4 & & $A C G$ & 0 & 0 & 2 & 0 & & $A A G$ & 4 & 7 & 3 & 4 & & $A G G$ & 0 & 0 & 0 & 0 \\
\hline \multirow[t]{4}{*}{ Val } & GUU & 0 & 1 & 0 & 1 & Ala & GCU & 2 & 1 & 2 & 1 & Asp & GAU & 1 & 0 & 1 & 0 & Gly & GGU & 0 & 2 & 2 & 2 \\
\hline & $G U C$ & 1 & 2 & 0 & 1 & & $G C C$ & 2 & 2 & 1 & 5 & & $G A C$ & 0 & 4 & 0 & 1 & & $G G C$ & 15 & 7 & 9 & 7 \\
\hline & GUA & 0 & 0 & 0 & 0 & & GCA & 0 & 0 & 1 & 3 & Glu & GAA & 0 & 1 & 1 & 0 & & GGA & 3 & 0 & 4 & 7 \\
\hline & $G U G$ & 2 & 1 & 1 & 1 & & $G C G$ & 2 & 0 & 1 & 0 & & $G A G$ & 4 & 5 & 3 & 6 & & GGG & 0 & 0 & 2 & 2 \\
\hline
\end{tabular}

Codon usage of W108 is shown.

Preferred codons in rice (Kawabe and Miyashita 2003) are shown in italic letter.

Table 5 Codon usage bias in the four metallothionein genes in O. rufipogon

\begin{tabular}{lllcccc}
\hline \hline Gene & \# codons & ENC & $(\mathrm{GC})_{\mathrm{t}}$ & $(\mathrm{GC})_{\mathrm{c}}$ & $(\mathrm{GC})_{3}$ & $\%$ Pref. \\
\hline met1 & 84 & 32.86 & 0.48 & 0.66 & 0.85 & 86.3 \\
met2 & 62 & 30.62 & 0.38 & 0.61 & 0.86 & 88.5 \\
met5 & 80.13 & 48.10 & 0.52 & 0.64 & 0.81 & 79.5 \\
met6 & 82 & 44.79 & 0.41 & 0.65 & 0.77 & 74.4 \\
\hline
\end{tabular}

ENC: effective number of codons.

$(\mathrm{GC})_{\mathrm{t}}: \mathrm{G}+\mathrm{C}$ content in the entire region, $(\mathrm{GC})_{\mathrm{c}}$ : in the coding region.

$(\mathrm{GC})_{3}$ : at the 3rd position, \%Pref: \% of preferred codons.

Table 6 Relationship between gene expression and DNA variation of methallothionein genes in Oryza rufipogon

\begin{tabular}{cccccc}
\hline \hline Gene & \multicolumn{1}{c}{$\%$ tag } & $\pi_{\text {total }}$ & $\pi_{\text {coding }}$ & $\pi_{\text {intron }}$ & Tajima's D \\
\hline met1 & $1.26(10.5)^{\mathrm{a}}$ & 0.0028 & 0.0019 & 0.0032 & $-0.88 \mathrm{NS}$ \\
met2 & $0.71(5.9)$ & 0.0101 & 0.0079 & 0.0118 & $-0.64 \mathrm{NS}$ \\
met5 & $0.17(1.4)$ & 0.0039 & 0.0035 & 0.0039 & $-1.62 \mathrm{NS}$ \\
met6 & $0.12(1.0)$ & 0.0051 & 0.0 & 0.0065 & $-1.22 \mathrm{NS}$
\end{tabular}

a: ratio to met6.

percentage of preferred codons. Although the association is not particularly high, codon bias (ENC) and nucleotide diversity for the entire region are also negatively correlated (Table 6).

It should be noted that a negative correlation between nucleotide diversity and level of gene expression was not observed when the coding region, but not introns, was considered, because the met6 coding region has no polymorphic sites. Also, the correlation between nucleotide variation in the entire region including introns and ENC in the coding region was unexpected. However, the observed relationship between nucleotide variation, gene expression and codon bias is in accordance with the working hypothesis mentioned above. Possible explanations for these results are discussed below.

Linkage disequilibrium in four $O$. rufipogon MT genes. A total of 1,035 combinations of DNA sequence variants were tested (Fig. 4), of which 192 pairs (18.6\%) showed significant nonrandom association at the $5 \%$ level. On average, $29.6 \%$ of the tests were significant in within-locus comparisons. In the met6 region, $40.9 \%$ of tested pairs were significant. These values are higher than in the O. rufipogon Adh1 locus (27.2\%; Yoshida et al., 2004). However, it is not possible to conclude whether these percentages of significant pairs in the MT regions are high, since linkage disequilibrium between DNA variations in nuclear genes of Oryza species has not been well investigated. Further studies are needed to answer this question.

In between-locus comparisons, an average of $12.9 \%$ of pairs was significant. Between the met2 and met6 regions, $21.8 \%$ were significant. This may be due to relatively small genomic distance between these two gene regions, which are $16.2 \mathrm{cM}$ apart on the short arm of the $1^{\text {st }}$ chromosome (Table 2). However, this percentage is 

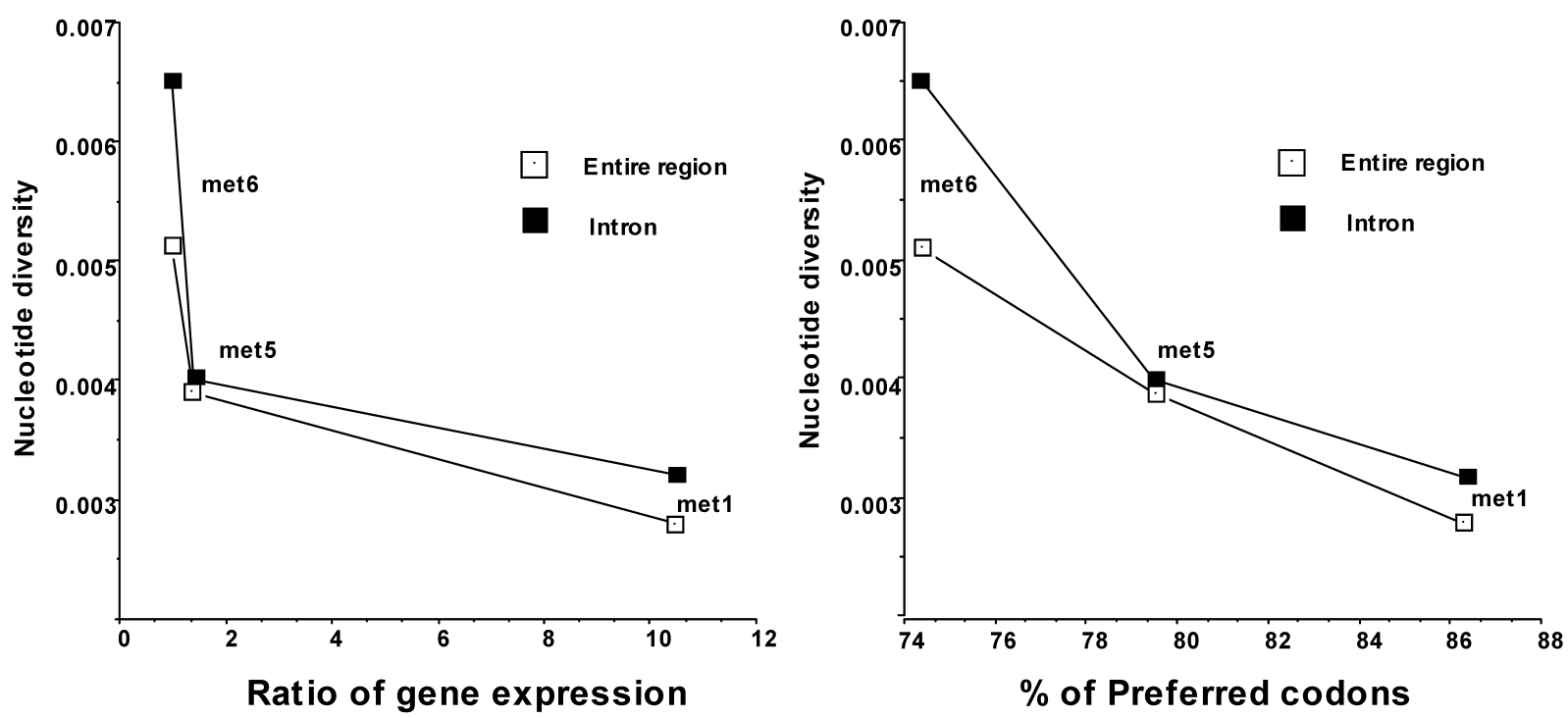

Fig. 3. Relationship between nucleotide diversity and level of gene expression (percentage of preferred codons) for type 2 MT genes. A) Ratio of gene expression relative to Met1 was used for the X-axis. B) Percentage of preferred codons was used for the Xaxis.

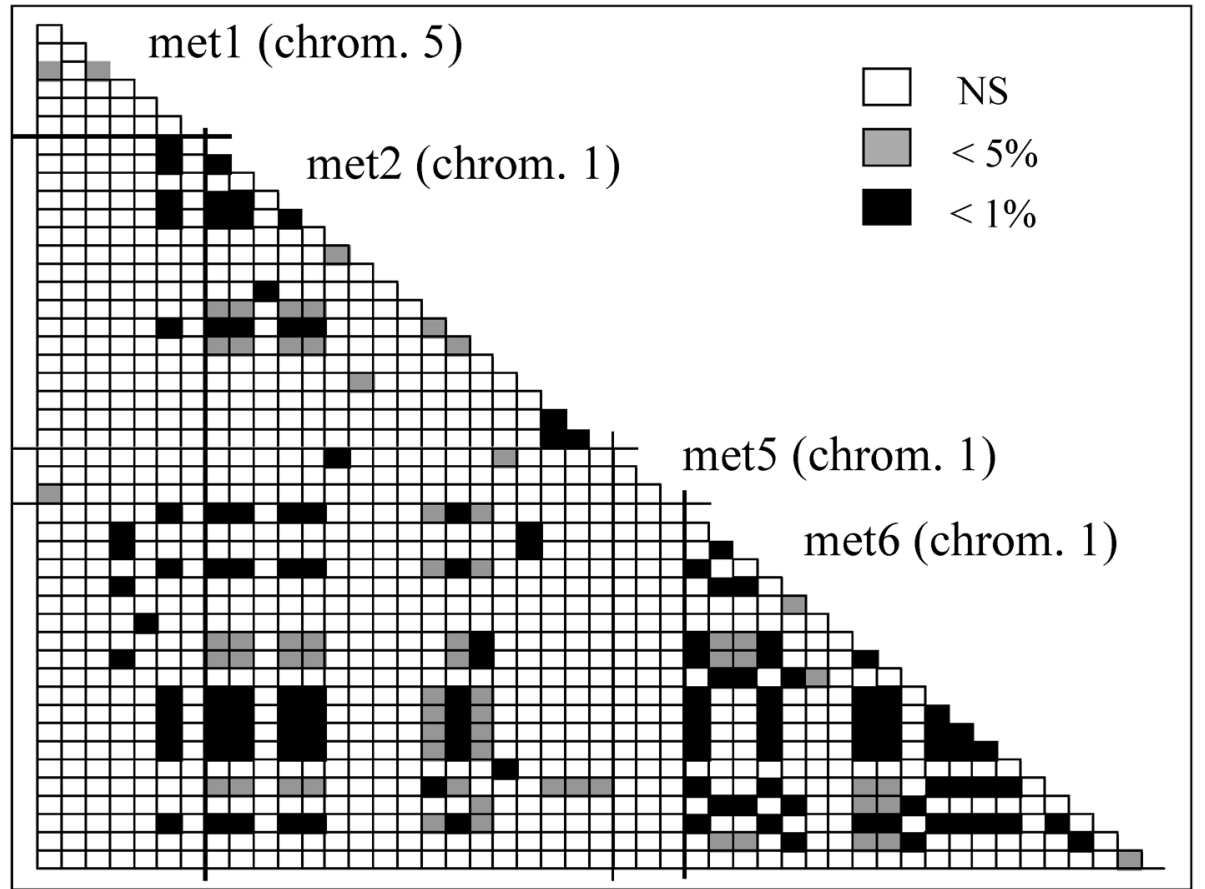

Fig. 4. Summary of linkage disequilibrium within and between MT genes of O. rufipogon. Informative DNA variations (both nucleotide sites and indels) detected from the 5' upstream region to the 3' end were arranged from top to bottom of the left side of the triangle. The four genes were delineated by thicker line. Significance of all the pairs of variations in the four genes examined by Chi-square test was shown.

higher than the within-locus value for met2 (19.1\%). In between-chromosome comparison, percentage of significant pairs between met1 (chromosome 1) and met6 (chromosome 5) was $8.6 \%$, which was close to the value for the met1 region $(9.5 \%)$. The percentage was not high (less than $5 \%$ ) in other comparisons.

It is notable that significant pairs are not distributed randomly (Fig. 4). In other words, particular variations are involved in significance both in within- and betweenlocus comparisons. This is mainly due to haplotype structure in the MT regions, especially in met2 and met6 (Fig. 2). Two accessions (W593 and W120) sampled from different regions have a distinct haplotype, sharing most polymorphic variations (site and indel) in the two 
regions. Significant linkage disequilibria were detected for pairs of these polymorphic variations, producing only two opposite gamete combinations out of four possible types in a $2 \times 2$ contingency table. Finally, it should be mentioned that after Bonferroni multiple-test correction, chi-square tests did not confirm significance for the data described above, probably due to lack of power caused by small sample size.

Phylogenetic relationship of $O$. rufipogon based on MT variation. As mentioned above, haplotype structure and epistatic interaction between gene family members influence linkage disequilibrium. But linkage disequilibrium can also be influenced by other factors including geographic population structure. To examine the latter possibility, neighbor-joining (NJ) trees were constructed for MT genes (Fig. 5). The topology of the $\mathrm{NJ}$ tree for each MT gene is similar to the topology of the parsimony tree (data not shown). Although different accessions of $O$. rufipogon were used for analysis of sequence variation in the four MT genes, it is clear that there is no association between clustering of accessions and geographic origin in either tree. In addition, the topology of the met5 NJ tree seems to be different from NJ trees for the other three MT genes, which have two large clusters. However, it is noted that accessions in the two clusters are not necessarily the same. These results indicate that population structure does not exist in $O$. rufipogon concerning the MT genes. Therefore, population structure probably does not greatly contribute to within- or between-locus linkage disequilibria for these genes.

In all trees, the cultivated rice $O$. sativa was included in the $O$. rufipogon cluster, because these species have a high level of sequence identity. The specific $O$. rufipogon accessions that are genetically closest to $O$. sativa vary from tree to tree, and depend on the sampling location (i.e., China, Indonesia or India). Thus, it is difficult to determine where $O$. sativa originated from this study.

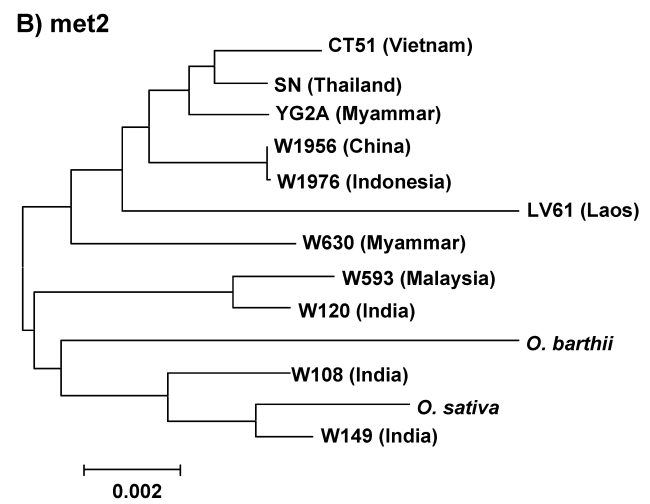

D) met6

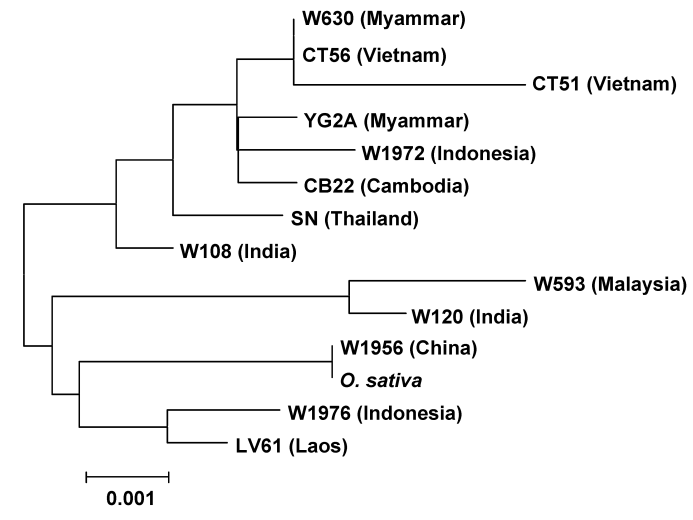

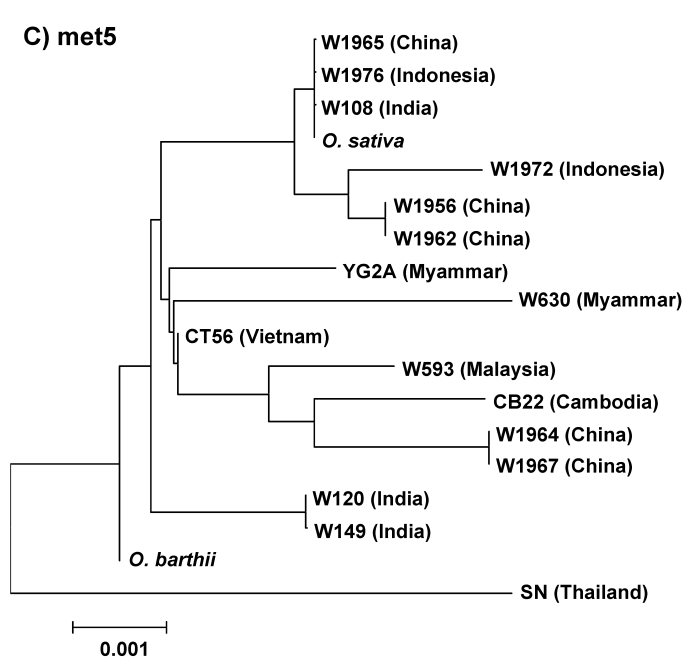

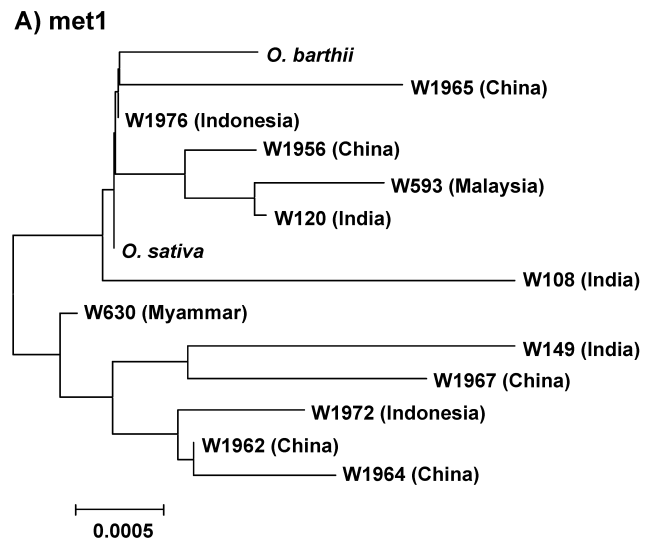

Fig. 5. NJ trees of Oryza species based on nucleotide variation in MT genes. A) A tree based on variation in the met1, B) met2, C) met5, and D) met6 genes, respectively. 


\section{DISCUSSION}

DNA polymorphism in O. rufipogon MT genes. Nucleotide variation was from 0.0028 and 0.0101 in the four O. rufipogon MT loci, which agrees with findings in other plant species and Drosophila. It is interesting to note this similarity in extent of nucleotide variation, which appears to be maintained in diverse organisms. Among the four $O$. rufipogon MT genes, met2 had the highest nucleotide variation, which led us to speculate that met2 is functionally distinct from the other three MT genes. Thus, it was excluded from some parts of our analysis (see below). It was also recently reported that met2 (Genbank accession \# AF001396) is highly expressed in blast-infected rice leaves (Matsumura et al. 2003), while the other three MT genes were not, which supports the hypothesis that met2 has a unique function within the MT gene family.

Clear-cut dimorphism (presence of two different sequence types) was not observed in the four O. rufipogon MT gene regions examined here, although this is a frequent property of nuclear genes in A. thaliana and was observed in the Adh3 region of wild barley (Hordeum vulgare ssp. spontaneum; Lin et al. 2001). However, met2 and met6 display notable haplotype structure, causing significant linkage disequilibria within and between loci. A common characteristic among these species is selfing nature of breeding system, which might have contributed to occurrence and maintenance of dimorphic haplotype structure. Further studies on these and other selfing species are needed to examine the relationship between breeding system and patterns of DNA polymorphism.

Relationship between nucleotide variation, gene expression and codon bias in $O$. rufipogon MT genes. This study confirms the hypothesis that gene expression is positively correlated with codon bias, providing evidence that this correlation occurs in four MT genes in $O$. rufipogon. Higher frequency of preferred codons in highly expressed MT genes than weakly expressed MT genes also supports this hypothesis. This correlation probably indicates natural selection at the translation level, and was previously suggested to reflect the availability of tRNAs in cells (Ikemura 1981; Moriyama and Powell 1997).

We also observed that gene expression correlated negatively with nucleotide variation in type 2 MT gene regions including introns, but not with nucleotide variation in the coding region of these genes. This correlation did not hold for met2, which was excluded from the analysis based on sequence divergence from the other MT genes. It is likely that this result reflects natural selection at the transcription level. Transcription is regulated by cis elements in the DNA promoter sequences $5^{\prime}$ to the coding region or alternatively by regulatory elements in intron DNA sequences. The latter possibility might explain the observation that intron DNA contributed strongly to the negative correlation between DNA variation and gene expression in these MT genes. Previous studies identified both positive and negative cis-acting elements in introns in genes from animal and plant species (Drosophila, Hinz et al. 1992; Gremke et al. 1993, maize, Callis et al. 1987; tobacco, Greschik et al. 1994, Arabidopsis, Sieburth and Meyerowitz 1997). It might not be unrealistic to assume the presence of enhancer(s) in intron(s) of these MT genes. This hypothesis implies that highly expressed genes require more stringent regulation than poorly expressed genes, so that level of nucleotide polymorphism in introns (and the entire region) in highly expressed genes would be lower than in poorly expressed genes, as observed here (Fig. 3). Additional studies are needed to identify regulatory elements in the 5 '-promoter or intron regions of these MT genes. If the above hypothesis is true, the correlation between nucleotide diversity in the entire gene region with codon bias (and percentage of preferred codons) might indicate that evolutionary mechanisms act coordinately on transcriptional and translational levels.

Linkage disequilibrium within and between $O$. rufipogon MT genes. Intergenic linkage disequilibrium was expected between the $O$. rufipogon MT genes, because they are epistatic and presumably coordinately regulated. This study demonstrated that there are significant linkage disequilibria not only within each MT gene, but between MT genes on the same or different chromosomes. Similarly, significant intragenic and interchromosomal linkage disequilibria were detected between two A. thaliana chitinase loci (ChiA and ChiB). The chitinase genes are both involved in defense against fungi, and in contrast, there was almost no significant linkage disequilibrium between $A d h$ and these two chitinase loci (Kawabe and Miyashita 1999). These results suggest that functional similarity between genes is related to significant intergenic (and inter-chromosomal) linkage disequilibrium. These results strongly support the idea that epistatic interaction related to coordinated gene regulation mechanisms contributes to significant linkage disequilibria.

Here, it was noted that haplotype structure also contributed to significant linkage disequilibria. However, there was no association between geographic origin of accessions and clustering in phylogenetic trees of MT genes. In fact, two accessions causing the haplotype structure were sampled in two different geographic locations. These results suggest that geographic structure is not a major factor related to linkage disequilibria in the $O$. rufipogon MT genes. Segregating variations (site and indel polymorphisms) in the two accessions, 
which were almost exclusively found in introns, might be involved in epistatic interactions. These variations might be related to enhancer elements discussed above. At this moment, the molecular mechanism of epistasis of these variations is not known. This study shows that it is useful to consider the relationship between gene expression and DNA polymorphism, and such studies can provide insight into the biological nature and significance of natural selection (and epistasis).

We express our thanks to R. Terauchi, S. Nasuda and Y. Matsuoka for their comments and suggestions on the early version of this manuscript. We would like to thank National Institute of Genetics, Japan, and Laboratory of Plant Breeding, Shizuoka University, Japan, for providing plant materials used in this study. Contribution number 584 from the Laboratory of Plant Genetics, Graduate School of Agriculture, Kyoto University.

\section{REFERENCES}

Akashi, H., and Schaeffer, S. W. (1997) Natural selection and the frequency distribution of "silent" DNA polymorphism in Drosophila. Genetics 146, 295-307.

Callis, J., Fromm, M., and Walbot, V. (1987) Introns increase gene expression in cultured maize cells. Genes Dev. 1, $1183-1200$

Cooper, G. M. (2000) The Cell. A Molecular Approach. Sinauer Associates, Inc. Sunderland, MA, USA

Fu, Y. X., and Li, W. S. (1993) Statistical tests of neutrality of mutations. Genetics 133, 693-709.

Genschik, P., Marbach, M., Uze, M., Feuerman, M., Plesse, B., and Storti, R. V. (1994) Structure and promoter activity of a stress and developmentally regulated polyubiquitin-encoding gene of Nicotiana tabacum. Gene 148, 195-202.

Grantham, R., Gautier, C., Gouy, M., Jacobzone, M., Mercier, R. (1981) Codon catalog usage is a genome strategy modulated for gene expressivity. Nucleic Acids Res. 9, r43-r74.

Gremke, L., Lord, P. C. W., Sabacan, L., Lin, S. C., Wohlwill, A., and Stroti, R. V. (1993) Coordinate regulation of Drosophila Tropomyosin gene expression is controlled by multiple muscle-type-specific positive and negative enhancer elements. Dev. Biol. 159, 513-527.

Hinz, U., Wolk, A., and Renkawitz-Pohl, R. (1992) Ultrabithorax is a regulator of b3 tubulin expression in the Drosophila visceral mesoderm. Development 116, 543-554.

Hsieh, H. M., Liu, W. K., and Huang, P. C. (1995) A novel stressinducible metallothionein-like gene from rice. Plant Mol. Biol. 28, 381-389.

Ikemura, T. (1981) Correlation between the abundance of Escherichia coli transfer RNAs and the occurrence of the respective codons in its protein genes. J. Mol. Evol. 146, 121.

Innan, H., Tajima, F., Terauchi, R., and Miyashita, N. T. (1996) Intragenic recombination in the Adh locus of the wild plant species Arabidopsis thaliana. Genetics 143, 1761-1770.

Jensen, L. G., Olsen, O., Kops, O., Wolf, N., Thomsen, K. K., and Von-Wettstein, D. (1996) Transgenic barley expressing protein-engineered thermostable (1,3-1,4)-beta-glucanase during germination. Proc. Natl. Acad. Sci. USA 93, 34873491.

Kägi, J. H. R., and Schäffer, A. (1988) Biochemistry of metallothionein. Biochemistry 27, 8509-8515.
Kawabe, A., and Miyashita, N. T. (1999) DNA variation in the basic chitinase locus $(\mathrm{ChiB})$ region of the wild plant Arabidopsis thaliana. Genetics 153, 1445-1453.

Kawabe, A., and Miyashita, N. T. (2003) Patterns of codon usage bias in three dicot and four monocot plant species. Genes Genet. Syst. 78, 343-352.

Kawashima, A. I., Kennedy, T. D., Chino, M., and Lane, B. G. (1992) Wheat Ec metallothionein genes. Eur. J. Biochem. 209, 971-976.

Kimura, M. (1956) A model of a genetic system which leads to closer linkage by natural selection. Evolution 10, 278-287.

Kimura, M. (1980) A simple method for estimating evolutionary rate of base substitutions through comparative studies of nucleotide sequences. J. Mol. Evol. 16, 111-120.

Kimura, M. (1983) The Neutral Theory of Molecular Evolution. Cambridge Univ. Press. Cambridge.

Kumar, S., Tamura, K., and Nei, M. (2001) MEGA2: Molecular Evolutionary Genetics Analysis software. Bioinformatics 17, 1244-1245.

Lin, J. Z., Brown, A. H. D., and Clegg, M. T. (2001) Heterogeneous geographic patterns of nucleotide sequence diversity between two alcohol dehydrogenase genes in wild barley (Hordeum vulgare subspecies spontaneum). Proc. Natl. Acad. Sci. USA 98, 531-536.

Matsumura, H., Nirasawa, S., and Terauchi, R. (1999) Transcript profiling in rice (Oryza sativa L.) seedlings using serial analysis of gene expression (SAGE). Plant J. 20, 719-726.

Matsumura, H., Stefanie, R., Ito, A., Saitoh, H., Kamoun, S., Winter, P., Kahül G., Reuter, M., Krüger, D. H., and Terauchi, R. (2003) Gene expression analysis of plant host-pathogen interactions by SuperSAGE. Proc. Natl. Acad. Sci. USA 100, 15718-15723.

Miyashita, N. T. (2003) Trimorphic DNA variation in the receptor-like protein kinase gene in the F18L15-130 region of the wild plant Arabidopsis thaliana. Genes Genet. Syst. 78, 221-227.

Morishima, H., Sano, Y., Oka, H. I. (1984) Differentiation of perennial and annual types due to habitat conditions in the wild rice Oryza perennis. Plant Syst. Evol. 144, 119-135.

Moriyama, E., and Hartl, D. L. (1993) Codon usage bias and base composition of nuclear genes in Drosophila. Genetics 134, 847-858.

Moriyama, E., and Powell, J. R. (1996) Intraspecific nuclear DNA variation in Drosophila. Mol. Biol. Evol. 13, 261-277.

Moriyama, E., and Powell, J. R. (1997) Codon usage bias and tRNA abundance in Drosophila. J. Mol. Evol. 45, 514-523.

Murphy, A., and Taiz, L. (1995) Comparison of metallothionein gene expression and nonprotein thiols in 10 Arabidopsis ecotypes. Correlation with copper tolerance. Plant Physiol. 109, 945-954.

Oka, H. I., and Chang, W. T. (1959) The impact of cultivation on populations of wild rice, Oryza $f$. spontanea. Phyton 13, $105-117$.

Okumura, N., Nishizawa, N. K., Umehara, Y., and Mori, S. (1991) An iron deficiency-specific cDNA from barley roots having two homologous cysteine-rich MT domains. Plant Mol. Biol. 17, 531-533.

Perlak, F. J., Fuchs, R. L., Dean, D. A., Mcpherson, S. L., and Fishhoff, D. A. (1991) Modification of the coding sequence enhances plant expression of insect control protein genes. Proc. Natl. Acad. Sci. USA 88, 3324-3328.

Robinson, N. J., Tommey, A. M., Kuske, C., and Jackson, P. J. (1993) Plant metallothioneins. Biochem. J. 295, 1-10.

Rozas, J., and Rozas, R. (1999) DnaSP version 3: an integrated 
program for molecular population genetics and molecular evolution analysis. Bioinformatics 15, 174-175.

Sieburth, J. E., and Meyerowitz, E. M. (1997) Molecular dissection of the AGAMOUS control region shows that cis elements for spatial regulation are located intragenically. The Plant Cell 9, 355-365.

Tajima, F. (1989) Statistical method for testing the neutral mutation hypothesis by DNA polymorphism. Genetics 123, 585-595.

Watterson, G. A. (1975) On the number of segregating sites in genetical models without recombination. Theor. Popul. Biol. 7, 256-276.
Wright, F. (1990) The "effective number of codons" used in a gene. Gene 87, 23-30.

Yoshida, K., Kamiya, T., Kawabe, A., and Miyashita, N. T. (2003) DNA polymorphism at the ACAULIS5 locus of the wild plant Arabidopsis thaliana. Genes Genet. Syst. 78, 11-21.

Yoshida, K., Ishii, T., and Miyashita, N. T. (2004) Nucleotide polymorphism in the Adh1 region of the wild rice Oryza rufipogon. Theor. Appl. Genet. 109, 1406-1416.

Zhou, J., and Goldsbrough, P. B. (1995) Structure, organization and expression of metallothionein gene family in Arabidopsis. Mol. Gen. Genet. 248, 318-328. 